\title{
Improvement of Corrosion Properties of AL-4.08MG Alloy in Seawater by Two Step Anodizing in Electrolyte with Varied Concentration
}

\author{
S.J. LEE ${ }^{a}$ AND S.J. KIM ${ }^{b, *}$ \\ ${ }^{a}$ Kunsan National University, Department of Power System Engineering, Daehak-ro 558, Gunsan-si, Jeonbuk, Korea \\ ${ }^{b}$ Mokpo National Maritime University, Division of Marine Engineering, Haeyangdaehak-Ro 91 Mokpo-si, Korea
}

\begin{abstract}
Aluminum is extremely active metal on the surface of which the oxide is instantly created when exposed to atmosphere. This naturally generated film has little industrial value as it has thickness as thin as $10 \mathrm{~nm}$. Hence, oxide films are often made thick by electrochemical method. The characteristics of the films can be controlled by varying process parameters including composition, concentration, additives, solution temperature, voltage etc. These films have high hardness and excellent corrosion resistance. Therefore, they are receiving great amount of attention in both academic and industrial areas with their diverse applicability. In this paper, the optimal electrolyte concentration was investigated to produce oxide film with excellent corrosion resistance. The results reveal that $\mathrm{Al}_{2} \mathrm{O}_{3}$ oxide film was created on the surface for all tested electrolyte concentrations. Moreover, the films have a considerably lower corrosion current density than that of the substrate, implying excellent corrosion resistance.
\end{abstract}

DOI: 10.12693/APhysPolA.129.742

PACS/topics: 75.70.-i, 82.45.Bb

\section{Introduction}

Aluminum oxide generated by electrochemical oxidation process is amorphous but it can be crystallized into $\alpha-\mathrm{Al}_{2} \mathrm{O}_{3}$ or $\gamma-\mathrm{Al}_{2} \mathrm{O}_{3}$ with heat treatment. This kind of oxide film layer consists of substrate aluminum, cells, pores and the barrier layer. The shape of the film is determined by voltage, temperature, electrolyte type, and electrolyte concentration during the anodizing process. The pores created on the film layer have sizes of tens to hundreds nanometers. The formation mechanism is often explained by the volume expansion that follows the oxidation reaction of aluminum [1]. The porous structure of aluminum oxide film was first analyzed by Keller in 1953, who explained that the oxide film has a structure, in which the pores are aligned in hexagonal shape [2]. This research has become very important in both chemical and physical fields. Diggle first proposed a model on the generation mechanism for the porous structure of the oxide film in 1968 [3]. Thompson and Wood attempted to clarify such formation mechanism using transmission electron microscopy [4]. Although a number of models explaining the pore creation process of aluminum oxide film have been continuously suggested, so far the mechanism of formation is still not clear. As for related literature, Wood et al. have reported that micro channel similar to a crack is created along the interior of the surface when anodizing by galvanostatic method and that it proceeds further following the anodizing to finally form a cylinder-shaped pore [5]. Moreover, Gösele et al.

*corresponding author; e-mail: ksj@mmu.ac.kr have reported that oxide film formation on the boundary interface of aluminum and electrolyte results in volume expansion, causing mechanical stress of surrounding pores [6]. In particular, Masuda and Fukuda proposed a two-step anodizing process for enhancing aluminum anodizing alignment in 1995 [7]. In the present study, we have performed surface modification through two-step anodizing process and evaluated electrochemical characteristics to improve corrosion resistance of aluminum alloy for marine service.

\section{Experimental method}

To fabricate a uniform porous film of 5083 aluminum alloy, we conducted electro-polishing under $25 \mathrm{~V}$ at $5{ }^{\circ} \mathrm{C}$ conditions for three minutes using mixed solution of ethanol (95\%) and perchloric acid (70\%) with volume ratio of $4: 1$. Afterward, the first step surface modification was performed using sulfuric acid as an electrolyte by controlling its concentration in the range from 5 to 20 vol.\%. For anode, 5083 aluminum alloy (Al-6.42Mg-0.4Si) with thickness of $5 \mathrm{~mm}$ and size of $2 \mathrm{~cm} \times 2 \mathrm{~cm}$ was used, while platinum electrode was used for cathode. The distance between the two electrodes was fixed at $3 \mathrm{~cm}$. To prevent hindrance of stable growth of oxidized layer due to local temperature increase during process, the electrolyte was agitated at $300 \mathrm{rpm}$ with a magnetic stirring. For anodizing, current density of $20 \mathrm{~mA} / \mathrm{cm}^{2}$ was applied between the anode and cathode for 40 minutes with electrolyte temperature controlled at $10^{\circ} \mathrm{C}$ in a jacketed beaker. For the second step surface modification process (identical to the step one), etching was performed using mixture of chromic acid $\left(1.8\right.$ wt.\%) and phosphoric acid $\left(6\right.$ wt.\%) at $60^{\circ} \mathrm{C}$ 
temperature for 30 minutes to remove the irregular oxide film that was created in the first step. After surface modification, pore structure was characterized using field emission scanning electron microscopy. The elemental analysis for surface was performed using energy dispersive spectroscopy, and the crystalline structure was identified using X-ray diffraction. Anodic polarization experiment was performed at scan rate of $2 \mathrm{mV} / \mathrm{s}$ up to $+3.0 \mathrm{~V}$ vs open circuit potential in natural seawater. Surface morphology was compared using 3D analysis microscope to observe the different damage characteristics with varied electrolyte concentration. Moreover, Tafel analysis was conducted by polarizing $\pm 0.25 \mathrm{~V}$ with an open circuit potential in the natural seawater to determine the corrosion potential and corrosion current density.

\section{Results and discussion}

Figure 1 shows different surface morphologies and porosities (the proportion of the area occupied by pores and the total area) after anodizing at different concentrations of sulfuric acid. The largest diameter of pores was in the case of electrolyte concentration of 5 vol.\% (Fig. 1a), although with a poor alignment of the barrier layer. In the case of $10 \mathrm{vol} . \%$, hexagonal structure that is unique to aluminum oxide film was clearly observed and the barrier layer was also well formed, however showing a smaller pore diameter, compared to the case of 5 vol. $\%$. On the other hand, in the case of 15 vol.\% and 20 vol.\%, rather irregular structure was observed along with small pores. In 1953, Keller has explained that aluminum oxide's pores are aligned in a hexagonal structure [2] and Diggle, afterwards, first proposed a model on their generation mechanism [3].

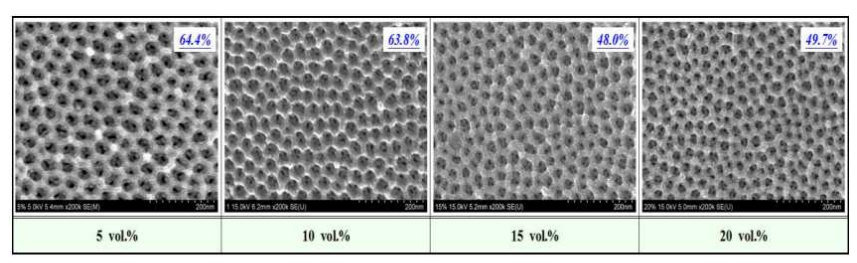

(a)
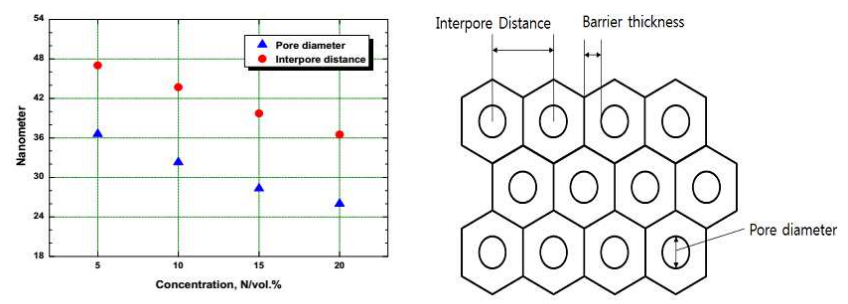

Fig. 1. Porosity analysis (a) and pore diameter and interpore distance (b) after AAO process for different sulfuric acid concentrations.

As $\mathrm{Al}_{2} \mathrm{O}_{3}$ is created on the boundary interface between aluminum and electrolyte, volume expands and mechanical stress in concentrated pores consequently occurs. Moreover, since this reaction simultaneously takes place in every pore, a repulsive force between pores appears. As a result, a barrier layer around the pores gets pushed upward, expanding vertically and forming the pore shape [2]. In this study, the porosity was determined to be $64.4 \%, 63.8 \%, 48.0 \%$, and $49.7 \%$ under the condition of electrolyte concentration of 5 vol. $\%$, 10 vol. $\%, 15$ vol.\%, and 20 vol.\%, respectively. The difference in porosity is resulted from the different reactivity, changing with the electrolyte concentration. It is considered that under the same conditions, as the electrolyte concentration increases, the solubility improves, making the previously formed barrier layer relatively thinner and resulting in more pores. With high electrolyte concentration, therefore, the area of the barrier layer increases with the increasing number of pores, which results in relatively lower porosity, compared to that of low-concentration electrolyte. Pore diameter as shown in Fig. $1 b$ was $36.3 \mathrm{~nm}, 32.3 \mathrm{~nm}, 28.3 \mathrm{~nm}$, and $26.0 \mathrm{~nm}$ at 5 vol.\%, 10 vol.\%, 15 vol.\%, and 20 vol.\%, respectively, indicating smaller pores at higher electrolyte concentrations. The same effects were obtained in the case where the created film gets exposed to electrolyte for a long time, because of relatively prolonged reaction time due to low solubility at low concentration. It is considered that barrier layer surrounding pores has dissolved, resulting in larger pore diameter. On the contrary, at high concentration, high solubility reduces reaction time to make the diameter smaller. Distance between pores which was $47.0 \mathrm{~nm}$ at $5 \mathrm{vol} . \%$ also decreased by $22 \%$ to $36.5 \mathrm{~nm}$ at 20 vol.\%. As the breakdown of barrier layer by negative ions in the electrolyte takes place in the early reaction, during the pore creation process, oxide thickness decreases. The electrolyte temperature is increased with the density of the current. Due to this, electrolyte activation increases to promote dissolution of the oxide film. Porous layer grows through this kind of chain reaction. Under the same conditions, concentrated current amount increases when electrolyte concentration is low, increasing temperature to activate, this in turn forms larger pore diameter.

Figure 2 depicts XRD analysis results for anodized surfaces obtained at different concentrations of sulfuric acid.

Diffraction peaks were observed only at $38^{\circ}, 44^{\circ}, 64^{\circ}$, and $77^{\circ}$, indicating no $\alpha$-alumina or $\gamma$-alumina peaks. Only peaks of the Al structure were observed. According to previous studies, $\mathrm{Al}$ alloy oxide film is said to be mainly composed of $\alpha$-alumina and $\gamma$-alumina [8]. However, it turned out that no crystalline structure was observed under low applied voltage conditions or without hydration treatment [9]. Based on the fact that only $\mathrm{Al}$ peaks have appeared for all experimental conditions, it is concluded that the oxide film did not experience a phase transformation, maintaining an initial amorphous state, regardless of the electrolyte concentration.

Figure 3 represents voltage-time response during anodizing process for different sulfuric acid concentrations. 


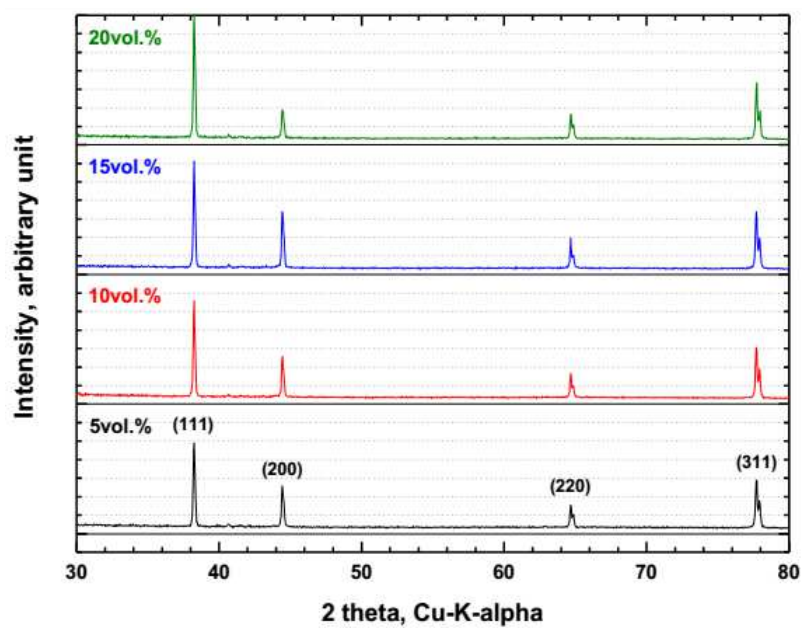

Fig. 2. XRD spectra after AAO process for different sulfuric acid concentrations.

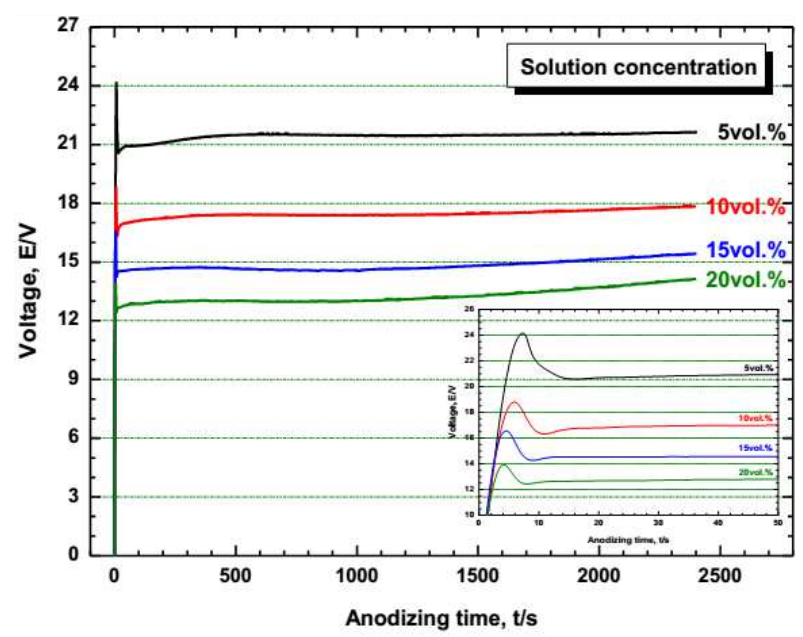

Fig. 3. Electrolysis voltage analysis for AAO process for different sulfuric acid concentrations.

With the increase of anodization time, the electrolysis voltage sharply increases from the very beginning and then decreased to a stable value. At the initial stage, with the sharp increase of electrolysis voltage, the electropolishing effect takes place due to dissolution of the surface. Therefore, a barrier layer was formed, while pores did not grow, until reaching the maximum electrolysis voltage. It is considered that as pores began to be created, the electrolysis voltage has decreased, and the stable behavior was observed by active migration of cations and anions. Moreover, a slight increase of electrolysis voltage at later stages implies the growth of porous film layer with the oxidation reaction. As a result, the final electrolysis voltage got lower with the increasing electrolyte concentration, with its value being $17.8 \mathrm{~V}$ (10 vol.\%), $15.3 \mathrm{~V}$ (15 vol.\%), and $14.2 \mathrm{~V}$ (20 vol.\%), all of which are lower than $21.6 \mathrm{~V}$ at 5 vol.\%. High electrolysis voltage means larger energy consumption required for anodizing, since high electrolysis voltage acts as a resistance to anodizing reaction. This in turn induces temperature increase by accelerating the electrochemical dissolution reaction, causing larger pore diameter. On the other hand, if electrolyte concentration increases, solubility also increases, making the previously formed barrier layer relatively thick. This promotes formation of pores and results in low electrolysis voltage. Therefore, more pores can be created within a short anodizing time in the case of high electrolyte concentration. This is consistent with the theoretical result, that pore diameter is proportional to the level of electrolysis voltage [10]. Following the same principle, the time necessary for reaching the maximum electrolysis voltage also decreases, with $7.28 \mathrm{~s}, 5.84 \mathrm{~s}, 4.59 \mathrm{~s}$, and $4.19 \mathrm{~s}$ at $5 \mathrm{vol} . \%, 10 \mathrm{vol} . \%$, 15 vol. $\%$, and 20 vol. $\%$, respectively. This implies that the pore formation time can be reduced with an increase in electrolyte concentration.

Figure 4 compares the anodic polarization test results in sea water after anodizing at different concentrations of sulfuric acid.

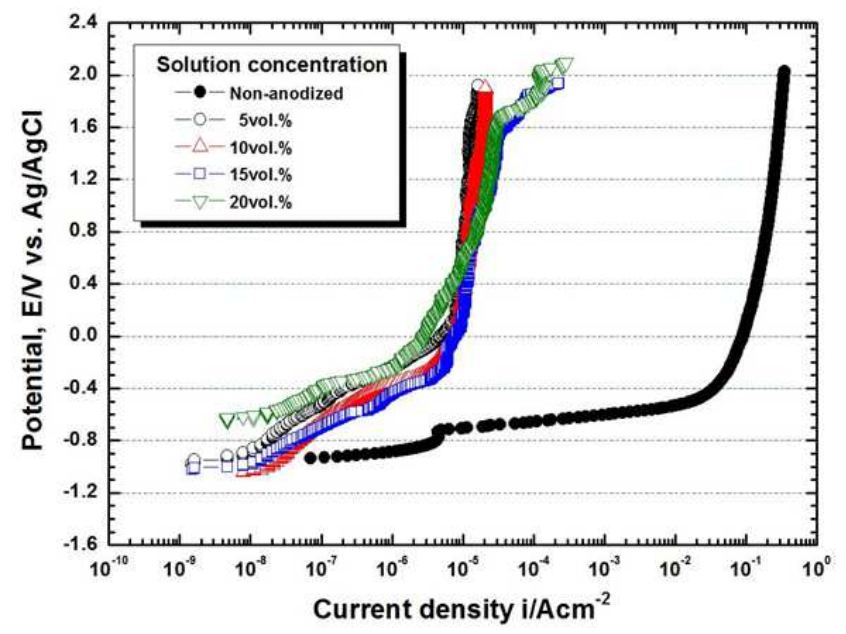

Fig. 4. Anodic polarization curves for AAO specimen for different sulfuric acid concentrations.

In overall, a considerably lower current density was observed over all range for the anodized specimens, compared with the non-anodized one. Without the anodizing treatment, the current density increased with the increasing potential at open circuit potential up to $4.61 \times 10^{-6} \mathrm{~A} / \mathrm{cm}^{2}$ and then a transient decrease was observed. Afterward, a dramatic increase in current density was observed due to pitting corrosion, which can be attributed to the activation of dissolution reaction on the surface due to excessive polarization. The lower current density in anodic polarization behavior is caused by the corrosion products created from the oxidation reaction, which suppress the movement of electric charge [11]. However, as the corrosion products are removed by the oxygen generated from the continued dissolution reaction and delamination, the current density begins to increase again. In the case of the non-anodized specimen, passivity was observed but its range was very narrow. Moreover, a dramatic increase of current density was observed 
above the pitting potential. For the anodized specimens, on the other hand, a wide range of passivity was observed, with low current density until the end of the polarization. Under the seawater immersion, aluminum alloy instantly forms a protective film such as $\mathrm{Al}_{2} \mathrm{O}_{3}$, but it can be damaged easily by chlorine ions in the seawater, causing a drastic increase of current density. However, the $\mathrm{Al}_{2} \mathrm{O}_{3}$ generated by the anodizing has a higher chemical stability and is thicker than the passive film that is created from the contact with the seawater. Therefore, it was possible to maintain low current density until the end of the experiment. In particular, a sharp increase in current density was observed over $1.6 \mathrm{~V}$ at 15 vol. $\%$ and 20 vol. $\%$. This may be attributed to the fact that excessive heat is generated at anodizing in a high-concentration electrolyte and a considerable difference in expansion coefficient between the substrate and the oxide film, as large as five times difference, results in micro cracks. Even though those cracks had no effect at low potential values due to weak dissolution reaction, it is assumed that current became concentrated at high potential to show a dramatic increase in current density. According to the anodic polarization test results, excellent electrochemical characteristics were observed in anodized samples due to the chemical stability of oxide with excellent corrosion resisting characteristics, compared to the sample which was not anodized.

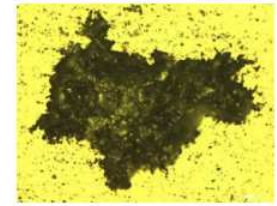

(a) Non-anodized

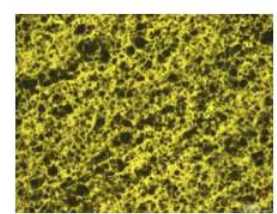

(d) 15 vol. $\%$

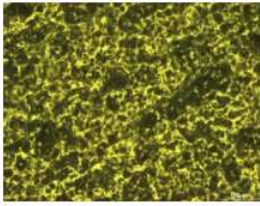

(b) 5 vol. \%

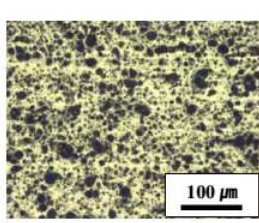

(e) 20 vol.\%

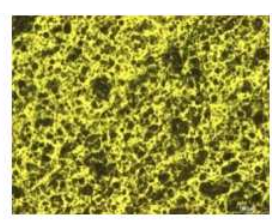

(c) 10 vol. $\%$

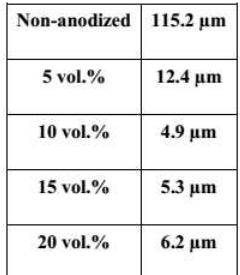

(f) Damage depth
Fig. 5. Comparison of surface analysis and damage depth after anodic polarization experiment for AAO at different sulfuric acid concentrations.

Figure 5 shows surface analysis results of anodic polarization in sea water of specimens anodized at different sulfuric acid concentrations. In case of non-anodized sample (Fig. 5a), a remarkably large surface damage was observed, which is due to corrosion and elimination phenomenon, due to sustained active state. In general, damage of aluminum hardly occurs because of the oxide film formation by the oxidation reaction during the early anodic polarization. However, since the oxide was removed due to sustained active state, pitting corrosion of $115.2 \mu \mathrm{m}$ in depth (Fig. 5f) was observed in local region. On the other hand, when anodizing was applied to samples, no difference of damage for different anodizing electrolyte concentrations was observed. In particular, a drastic increase of current density was observed on polarization curve before the termination of polarization for $15 \mathrm{vol} . \%$ and $20 \mathrm{vol} . \%$ samples. However, this is the concentration of current through micro cracks in a short period of time and thus no effect of actual damage was observed in Figs. 5d and e. In anodized samples the measurement results of damage depth indicate largest damage depth of $12.4 \mu \mathrm{m}$ at 5 vol.\% (Fig. 5b) and smallest damage depth of $4.9 \mu \mathrm{m}$ at $10 \mathrm{vol} . \%$ (Fig. $5 \mathrm{f}$ ). The damage depth showed similar values for different anodizing electrolyte concentrations. This can be interpreted as follows, even though the diameter of created pores differs with the electrolyte concentration, they have identical elements due to the characteristics of the electrochemical experiment that depend on the surface element. Hence no large difference was observed. A large difference in damage amount between the anodized and non-anodized is assumed to be caused by the presence of $\mathrm{Al}_{2} \mathrm{O}_{3}$ on the surface. The $\mathrm{Al}_{2} \mathrm{O}_{3}$ has high electric insulation resistance, excellent durability to abrasion, and chemical stability. Since it impedes movement of electric charge, it has superior electrochemical characteristics.

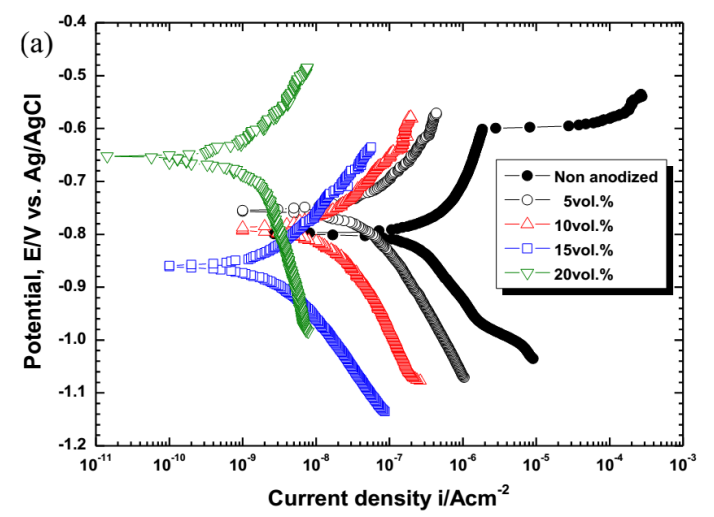

(b)

\begin{tabular}{|c||c|c|}
\hline Concentration & $\begin{array}{c}\text { Corrosion } \\
\text { potential }\end{array}$ & Corrosion \\
\hline \hline As-received & $-0.779 \mathrm{~V}$ & $2.09 \times 10^{-7} \mathrm{~A} / \mathrm{cm}^{2}$ \\
\hline 5 vol.\% & $-0.755 \mathrm{~V}$ & $6.16 \times 10^{-8} \mathrm{~A} / \mathrm{cm}^{2}$ \\
\hline 10 vol.\% & $-0.790 \mathrm{~V}$ & $2.29 \times 10^{-8} \mathrm{~A} / \mathrm{cm}^{2}$ \\
\hline 15 vol. $\%$ & $-0.858 \mathrm{~V}$ & $2.94 \times 10^{-9} \mathrm{~A} / \mathrm{cm}^{2}$ \\
\hline 20 vol. $\%$ & $-0.651 \mathrm{~V}$ & $1.53 \times 10^{-9} \mathrm{~A} / \mathrm{cm}^{2}$ \\
\hline
\end{tabular}

Fig. 6. Polarization curves for Tafel analysis (a) and analysis results (b) for AAO specimens obtained using different sulfuric acid concentrations. 
Figure 6 depicts Tafel analysis results of samples anodized at different sulfuric acid concentrations. The polarization curves for Tafel analysis are shown in Fig. 6a. Concentration polarization phenomenon was observed in cathodic polarization curve in the non-anodized sample and current density has increased with potential shift towards active direction. Moreover, anodic polarization curve showed that current density had gradually increased with increasing potential and drastically increased at $-0.601 \mathrm{~V}$ due to appearance of pitting corrosion, where the current density was approximately $1.85 \times 10^{-6} \mathrm{~A} / \mathrm{cm}^{2}$. On the other hand, in case of anodized samples, anodic polarization and cathodic polarization proceeded to increase the potential difference from the open circuit potential and a gradual increase of current density was observed. When anodizing was applied, a similar trend was observed in overall. This is because it greatly depends on the chemical composition of the surface, since polarization is induced within narrow potential region of $\pm 250 \mathrm{mV}$, which has a scarce corrosion damage. Therefore, the oxide created on the surface is identical to $\mathrm{Al}_{2} \mathrm{O}_{3}$ and the intensity of anodic and cathodic polarization with open circuit potential is small. Therefore, the current density has turned out to be very stable and relatively low, compared to the case without anodization. According to the corrosion potential measurement results, electrolyte concentration at 20 vol.\% that showed most noble corrosion potential, turned out to be electrochemically most stable as well, whereas 15 vol. $\%$ with lowest corrosion potential turned out to be the worst. In general, the corrosion potential in neutral solution is formed by concentration polarization according to the diffusion of dissolved oxygen and active polarization by oxidation reaction on the anode. When determining corrosion rate, corrosion current density is assumed to be the diffusion limiting current density on the polarization curve. According to the corrosion current density comparison results in Fig. 6b, corrosion current density was high at $2.09 \times 10^{-7} \mathrm{~A} / \mathrm{cm}^{2}$ in case without anodization. However, in the anodized samples, very low corrosion current density was observed with its value of $6.16 \times 10^{-8} \mathrm{~A} / \mathrm{cm}^{2}, 2.29 \times 10^{-8} \mathrm{~A} / \mathrm{cm}^{2}$, $2.94 \times 10^{-9} \mathrm{~A} / \mathrm{cm}^{2}$, respectively. This indicates a significant improvement of corrosion resistance in seawater. In overall, corrosion current density turned out to continuously decrease with the increase in electrolyte concentration. This can be interpreted as follows, the porosity has decreased with the increase in electrolyte concentration and the area of $\mathrm{Al}_{2} \mathrm{O}_{3}$ on the surface has relatively increased, giving rise to low corrosion current density. Therefore, the results indicate an increase of lifetime following the decreased attrition rate because of slower dissolution rate, compared to the case without anodizing.

\section{Conclusions}

In this study, electrochemical evaluations were carried out to determine the optimal sulfuric acid concentration for anodizing, for achieving optimal corrosion resistance in sea water. The surface observation results indicate different reactivity with electrolyte concentration, showing largest pore at $5 \mathrm{vol} . \%$ and highest alignment at $10 \mathrm{vol} . \%$. In the electrochemical experiment, the anodized specimens showed considerably low corrosion current density than that of the non-anodized specimen, implying excellent corrosion resistance. However, attention is to be paid in the case of high electrolyte concentration since micro cracks can appear. In conclusion, 10 vol. $\%$ that presented least damage is believed to the optimal sulfuric acid concentration.

\section{Acknowledgments}

This research was a part of the project titled "Construction of eco-friendly $\mathrm{Al}$ ship with painting, and maintenance/repairment free", funded by the Ministry of Oceans and Fisheries, Korea.

\section{References}

[1] H. Masuda, F. Hasegwa, S. Ono, J. Electrochem. Soc. 144, L127 (1997).

[2] F. Keller, M.S. Hunter, D.L. Robinson, J. Electrochem. Soc. 100, 411 (1953).

[3] J.W. Diggle, T.C. Downie, C.W. Goulding, Chem. Rev. 69, 365 (1969).

[4] G.C. Wood, P. Skeldon, G.E. Thompson, K. Shimizu, J. Electrochem. Soc. 143, 74 (1996).

[5] Y. Xu, G.E. Thomson, G.C. Wood, Trans. Inst. Met. Finish. 63, 98 (1985).

[6] A. Li, F. Muller, A. Bimer, K. Nielsch, U. Gosele, J. Appl. Phys. 84, 6023 (1998).

[7] H. Masuda, K. Fukuda, Science 268, 1466 (1995).

[8] X. Nie, E.I. Meltis, J.C. Jiang, A. Leyland, A.L. Yerokin, A. Matthews, Surf. Coat. Technol. 149, 245 (2002).

[9] E.K. Joo, Ph.D Thesis, Kookmin University, 2002 (in Korean).

[10] T.P. Hoar, J. Yahalom, J. Electrochem. Soc. 110, 614 (1963).

[11] M. Pourbaix, Atlas of Electrochemical Equilibria in Aqueous Solutions, National Association of Corrosion Engineers, Houston 1974. 